\title{
Mucociliary Clearance as an Outcome Measure for Cystic Fibrosis Clinical Research
}

\author{
Scott H. Donaldson', Timothy E. Corcoran², Beth L. Laube', and William D. Bennett ${ }^{4}$ \\ 'Department of Medicine, University of North Carolina, Chapel Hill, North Carolina; ${ }^{2}$ Departments of Medicine and Bioengineering, University \\ of Pittsburgh, Pittsburgh, Pennsylvania; ${ }^{3}$ Department of Pediatrics, The Johns Hopkins University School of Medicine, Baltimore, Maryland; \\ and ${ }^{4}$ Center for Environmental Medicine and Lung Biology, University of North Carolina at Chapel Hill, Chapel Hill, North Carolina
}

\begin{abstract}
Current concepts of cystic fibrosis (CF) pathophysiology link ion transport abnormalities to reduced airway surface liquid (ASL) hydration and impaired mucus clearance. It is likely that correction of the defects that cause ASL dehydration will prevent degradation of mucus clearance, thereby preventing the initiation and/or progression of CF lung disease. A number of novel therapeutic agents aimed at the earliest steps in disease pathogenesis are now under development for the treatment of CF lung disease. Consequently, there is a tremendous need to develop methods that directly assess the effects of these agents on the underlying pathophysiologic process in the target organ. The measurement of mucociliary clearance (MCC) is a highly biologically relevant outcome, but one that is in need of further development. Here, we describe important methodologic aspects of MCC measurement and issues that have limited its use as an outcome measure in the past. Furthermore, we outline the steps that are being carried out now, and will be carried out in the future, to improve the performance of these studies in clinical trials. A systematic approach to optimizing and standardizing the measurement of MCC should greatly advance our ability to assess novel therapies at a relatively early stage of drug development. The resulting data may then be used to select those candidates that should be rapidly advanced into larger clinical trials.
\end{abstract}

Keywords: cystic fibrosis; mucociliary clearance; mucus clearance; scintigraphy

The pathogenesis of cystic fibrosis (CF) lung disease is complex and involves a progression of events that lead to chronic airway infection and bronchiectasis. The dominant view of early disease pathogenesis links epithelial ion transport abnormalities (see article by Rowe and colleagues in this symposium [pp. 387-398] [1]) to dehydration of the airway surface liquid (ASL) layer (2). A normally hydrated ASL layer, which includes both a lowviscosity periciliary layer (PCL) and an overlying mucus layer, is required for optimal mucus transport in the lung (3). Mucus dehydration dramatically alters its transportability while also making it impervious to neutrophil influx, thus limiting their ability to reach their bacterial targets (4). Volume depletion of the PCL interferes with normal cilia motion and ultimately allows contact and adhesion between the mucus layer and epithelial cell surfaces. Together, these defects degrade cilia and coughdependent mucus clearance, which comprise the primary innate defense mechanism for the lung (5). In CF, defective mucus clearance is proposed to be an integral step in disease pathogenesis, and is the target of a number of drugs currently in the

(Received in original form March 15, 2006; accepted in final form April 25, 2007) Supported by the Cystic Fibrosis Foundation.

Correspondence and requests for reprints should be addressed to Scott $\mathrm{H}$. Donaldson, M.D., Cystic Fibrosis Research and Treatment Center, 6007B Thurston Bowles Building, CB\#7248, University of North Carolina at Chapel Hill, Chapel Hill, NC 27599. E-mail: scott_donaldson@med.unc.edu

Proc Am Thorac Soc Vol 4. pp 399-405, 2007

DOI: $10.1513 /$ pats.200703-042BR

Internet address: www.atsjournals.org development pipeline. A sensitive and specific outcome measure that directly reflects the mucus clearance defect in CF as well as the impact of effective therapies would, therefore, be an invaluable tool for the drug development process.

The measurement of mucociliary clearance (MCC) has, in fact, been performed for decades via various techniques. The inhalation of aerosolized, radiolabeled particles is the most widely accepted technique. However, significant variation exists in the nature of the inhaled particles, their delivery to the airway, and the analysis and interpretation of MCC studies, and has limited its acceptance as a reliable outcome measure. The ongoing development of CF therapies aimed at normalizing ASL hydration and mucus clearance makes refinement and validation of the MCC measurement technique an important priority for the $\mathrm{CF}$ research community. The ensuing text describes the current status of MCC measurements and further outlines the necessary refinements that will increase the utility of this outcome measure.

\section{TECHNICAL ASPECTS OF MCC MEASUREMENTS}

The measurement of MCC or cough clearance (CC) rate requires the delivery of an externally detectable and nonabsorbable marker to the airways. Past studies have utilized substances such as technetium-99m-labeled iron oxide and human serum albumin for this purpose. More recent studies have relied on the nebulized delivery of technetium sulfur colloid (Tc-SC) aerosols. The unfiltered form of Tc-SC contains submicronic particles (mean size, $0.3 \mu \mathrm{m}$ ) (6) in isotonic saline. This solution is then nebulized to form larger aerosol particles that are inhaled. After delivery to the airways, minimal tracer penetration into the bloodstream is observed, and the bulk of the Tc-SC mass is thought to remain suspended within the mucus layer (7). Luminal macrophages do, however, engulf a significant fraction of deposited particles within the time frame of a typical MCC study $(31 \%$ after $100 \mathrm{~min}$ ), but clearance of these macrophages is thought to occur at a similar rate to free Tc-SC particles (8).

The lung deposition pattern of aerosolized particles depends upon the characteristics of the aerosol particles (average size, distribution of size), the breathing pattern used during inhalation (flow rate, tidal volume), and the nature of the airways themselves (i.e., degree of obstruction and lung size) (9). This is critically important, because the observed rates of MCC vary in different lung regions, and, therefore, are highly dependent on the deposition pattern (10). For example, MCC rates within small airways are on the order of $1 \mathrm{~mm} /$ minute, whereas rates in large airways and the trachea may be as high as $2 \mathrm{~cm} /$ minute (11). Macrophage-mediated clearance of particulates deposited in the alveoli is much slower (i.e., months). Although the ability to manipulate aerosol characteristics and breathing patterns provides the opportunity to target and study different lung regions, the resulting variability in lung deposition also makes the generation of reproducible MCC data difficult. Therefore, an emphasis has been placed on devising aerosol delivery systems with a high degree of deposition pattern reproducibility, thereby reducing 
measurement error and increasing the ability to detect differences between groups. These systems typically use an aerosol generation device and a series of delivery adjuncts to produce repeatable breathing patterns and consistent aerosol dosing. For example, pneumotach devices with visual feedback are often used to control inhalation flow rate and tidal volume within specific ranges; metronomes can be used to guide the timing of inhalation and exhalation; and aerosol dosimetry equipment can be used to pulse aerosol delivery during specific portions of the breathing cycle.

Despite careful efforts to minimize the variability of aerosol deposition, significant differences that confound MCC data may still occur within a patient on consecutive studies or between groups of individuals. Therefore, it is very important that initial deposition patterns be quantified and factored into comparisons between studies. Deposition parameters are typically expressed as ratios of radioactive counts in central and peripheral lung zones made at the beginning of the clearance measurement period $(\mathrm{C} / \mathrm{P}$ ratio). In principle, peripheral zones will comprise primarily slowly clearing small airways, whereas central regions, although also containing the small airways, will be relatively enriched with more rapidly clearing large airways. To define these lung regions, either a transmission scan (using an external radiation source) or a ventilation scan (using an inhaled radioactive gas, such as xenon-133) must be performed so that the outer lung boundaries can be identified and regions of interest assigned (Figure 1A). Once identified, the initial aerosol deposition image (Figure 1B) may be used to quantitate the $\mathrm{C} / \mathrm{P}$ ratio. Clearance rates in each region (i.e., whole lung, central, peripheral) may then be expressed as a function of retained counts versus time. When a xenon-133 ventilation scan is used to define lung boundaries, the $\mathrm{C} / \mathrm{P}$ ratio can be further normalized to the lung volume contained within each lung zone using the counts measured after achieving equilibrium during xenon inhalation (12). Alternatively, deposition may be described using a parameter called the "penetration index" (PI). The PI is the ratio of radioactive counts per pixel area in the peripheral lung zone to counts per pixel area in the central zone (13). Because increased deposition in central airways, as indexed by a high $\mathrm{C} / \mathrm{P}$ ratio or low PI, is associated with increased rates of MCC, it is critical to account for this potential confounder in the analysis of these data (Figure 1D) (14). A different approach to circumventing errors that result from differences in aerosol deposition focuses on clearance measured in the peripheral lung zone (12). Because this region contains a somewhat more homogeneous composition of airways than the central or whole-lung regions, resulting clearance rates are expected to be less sensitive to the initial deposition pattern. This approach can be problematic, however, because reduced tracer deposition (with traditional delivery systems) and intrinsically slower clearance rates in this region lead to a smaller signal:noise ratio. Drug effects on central/larger airways may also be missed by this approach.

The temporal relationships between a particular intervention, the delivery of inhaled radiolabel, and the onset of imaging are also important considerations. This is particularly true because of the two-phase nature of clearance that is typically observed (Figure 1C) (7). A fast clearance phase is usually noted immediately after the administration of the Tc-SC aerosol, lasting for a period of 15-40 minutes. A slower clearance phase is then observed, which lasts through the rest of most typical imaging periods (i.e., 1-2 h). It is thought that the fast phase of clearance represents the initial, rapid transit of deposited particles from larger airways, whereas the slow phase reflects small airway clearance. It is possible, however, that slower clearance phases could reflect the movement of the radiolabel into a less clearable compartment (e.g., due to mixing into the PCL vs. residence in the mucus layer, or intracellular uptake vs. free Tc-SC label), rather than being a function of airway deposition. The time between aerosol administration and the commencement of imaging will affect the proportion of the fast phase that is sampled,
A
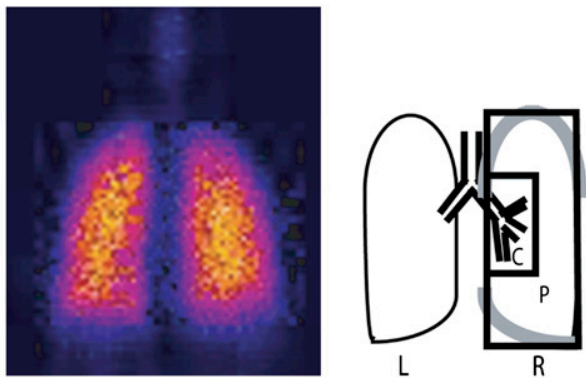

B
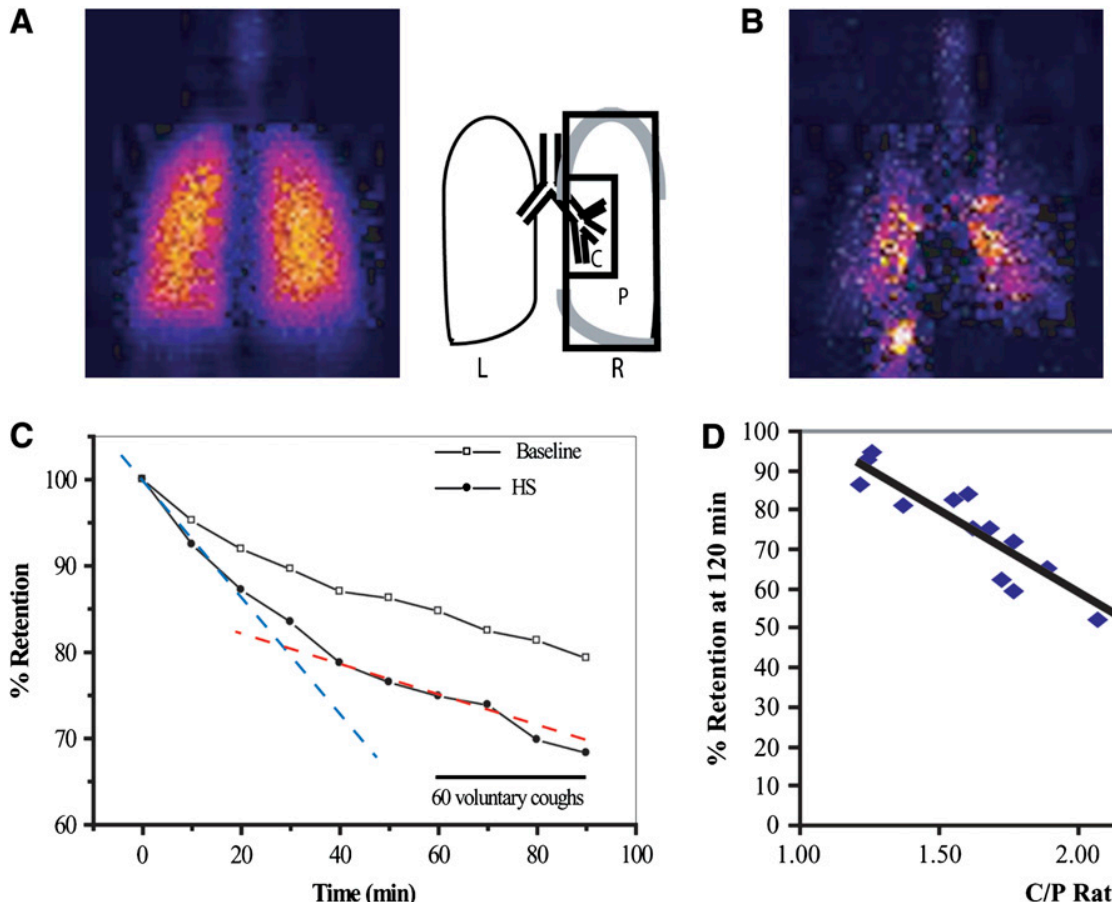

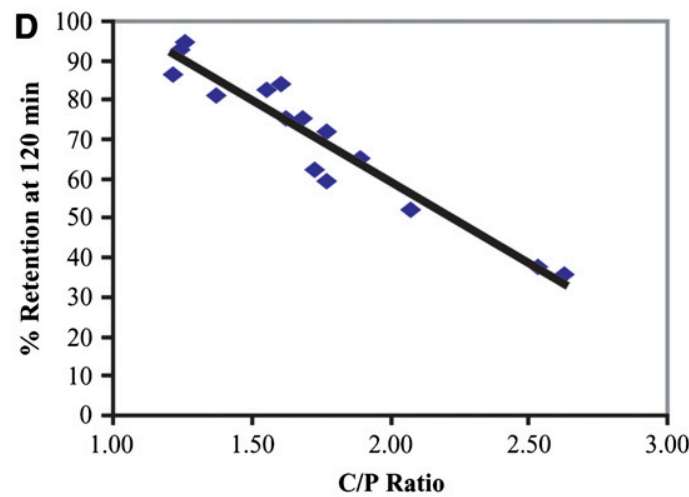

Figure 1. (A) A xenon-133 equilibrium scan is used to identify lung ( $L$, left; $R$, right) boundaries in a normal subject. Using this outline, central (C) and peripheral $(P)$ regions of interest are assigned. $(B)$ This deposition image was obtained immediately after the inhalation of technetium sulfur colloid in the same subject. (C) Mean rates of clearance from 12 subjects with cystic fibrosis are shown at baseline (open squares) and immediately after inhalation of hypertonic saline (HS) (16). The "fast phase" (through 20 min; blue dashed line), reflecting clearance from large airways, and "slow phase" (from $40 \mathrm{~min}$ to start of cough clearance measurement; red dashed line), reflecting smaller airway clearance, are highlighted on the post-HS clearance curve. (D) The effect of the ratio of radioactive counts measured in the

central and peripheral lung zones (C/P ratio) on rates of mucociliary clearance, as denoted by particle retention through 120 minutes, in a cohort of normal study subjects. Images in $(A)$ and $(B)$, and data in $(D)$ (previously unpublished) courtesy of W.D. Bennett. 
and could potentially have substantial effects on the overall measured rate. Comparative trials, therefore, must carefully match the time between Tc-SC delivery and the start of imaging.

Once subjects have inhaled the radiolabeled particles, they are positioned in front of a camera that detects and records emitted $\gamma$ radiation. The sensitivity and spatial resolution of the resulting image will be a function of the inhaled Tc-SC dose, the camera resolution (i.e., pixel matrix size), and the thickness of the associated collimator. Collimators are thin plates that are placed between the patient and the detecting surface of the $\gamma$ camera, which filter out "scatter," allowing only radiation coming from a direction perpendicular to the camera to reach the imaging surface (15). In general, camera properties that improve resolution (more pixels/inch, thicker collimator) also reduce its sensitivity, causing a trade-off that can only be compensated by increasing the administered Tc activity. Longer acquisition times for each $\gamma$ camera image will also improve the signal available for analysis while reducing the number of images (i.e., data points) that can be collected during the clearance measurement. Patient positioning during imaging is another factor that varies between centers, with some studies using seated positioning (16) and others using recumbent positioning (13). Seated positioning provides for easier aerosol delivery and, arguably, a more physiologically realistic assessment. Recumbent positioning may improve subject comfort and minimize movement during imaging. Differences in rates of clearance as a result of patient position have not been explored. Finally, images used in the calculation of MCC must be corrected for decay of the isotope and for background radiation level. Typically, only the right lung is analyzed, because activity in the stomach can bias left-lung counts.

MCC measurements are often supplemented by measurements of CC, and by extended (i.e., 24-h) measurements of clearance. CC is typically measured by having the subjects cough at prescribed frequencies through an expiratory flow measurement device to gauge the forcefulness of each cough. In most cases, a period of CC is included some time after the initial measurement of MCC $(16,17)$, although, in this configuration, the amount of isotope available in airways available to clearance by cough (i.e., more central airways) may be significantly reduced, thereby lowering the sensitivity of this measurement. Furthermore, because a preceding period of cilia-based clearance will have variably reduced the amount of isotope accessible to clearance by cough on different study days, a further normalization (e.g., C/P measured at start of coughs) must be used before comparing CC measurements. Current protocols for $\mathrm{CC}$ measurement in $\mathrm{CF}$, therefore, are suboptimal, because study designs used to measure this clearance parameter have prioritized it below cilia-dependent clearance measurements.

Measurements of 24-hour clearance have gained renewed interest recently, and serve several purposes. First, they provide an additional means of assessing aerosol deposition pattern, because aerosol initially delivered to the alveoli will be retained for a much longer period than aerosol delivered to the airways $(10,18)$. Second, these measurements can also be used to provide a measurement of cumulative clearance over longer time domains, which, in turn, may illustrate therapeutic efficacy (16). The relative contribution of (1) alveolar deposition, (2) slowphase clearance from peripheral airways, and (3) mixing into a poorly cleared compartment of the ASL to 24-hour measures of clearance remains unclear. In addition, because the half-life of technetium-99m is approximately 6 hours, assessment of 24hour clearance requires the delivery of sufficient initial tracer activity to ensure signal levels that are sufficiently above background at later timepoints.

\section{AS AN OUTCOME MEASURE IN CF}

Comparison of MCC measurements in healthy subjects and patients with $\mathrm{CF}$, as well as other accumulated experiences with MCC measurements in clinical trials, provides an opportunity to judge the ability of MCC measurement techniques to serve as a biomarker of disease and/or drug activity. In this section, we will review both positive and negative data pertinent to these issues, and will discuss important issues that should be addressed in future studies.

Several published comparisons of MCC measurements in unaffected individuals and subjects with $\mathrm{CF}$ are available for review. In the largest, by Robinson and colleagues, a retrospective review of one laboratory's experience in normal subjects $(\mathrm{n}=$ 17 subjects/22 studies) and subjects with CF ( $\mathrm{n}=59$ subjects/ 184 studies) was reported (13). Salient features from this study include the fact that mucus clearance was indeed markedly impaired in subjects with $\mathrm{CF}$ in comparison to the normal cohort. This reduction in clearance in patients with CF was most apparent in central and intermediate regions (vs. peripheral) and in apical and "mid" zones (vs. basal). In each of the most affected regions, clearance was approximately $50 \%$ that of the normal rate. Interestingly, the observed reductions in clearance appeared to be independent of baseline lung function, and even a subgroup of subjects with $\mathrm{CF}$ with normal $\mathrm{FEV}_{1}$ measurements $(\mathrm{n}=17)$ manifested markedly abnormal clearance rates. In other comparisons of clearance rates between unaffected individuals ( $\mathrm{n}=12-17$ each) and patients with $\mathrm{CF}$ with mild to moderate impairment in lung function ( $\mathrm{n}=12-20$ each), whole-lung clearance was, in fact, not different $(12,16)$. However, MCC indices that reflect clearance from small airways, particularly peripheral zone clearance and 24-hour clearance, were significantly reduced $(12,16,19)$. Finally, a study of long-term clearance from $\mathrm{CF}$ airways used a delivery technique that specifically targeted small airways with a radiolabeled particle that allowed detection of clearance over a 3-week period. This study demonstrated significantly increased particle retention after 24-hours in subjects with CF (67 vs. $48 \%$, CF vs. normal, respectively). Particle retention between 7 and 21 days was not affected. Although the authors contended that these data did not reflect a small airway clearance defect in CF, but, rather, reflected delayed clearance from large and medium-sized airways, this interpretation is debatable. In fact, because there was very little predicted large/medium-sized airway deposition with the inhalation technique used (20-25\% of total deposited fraction), it would appear likely that delayed small airway clearance did indeed contribute to the difference in 24-hour retentions between normal subjects and those with $\mathrm{CF}$. The lack of a difference between clearance after 7 days may signify that clearance mechanisms other than MCC (e.g., cellular uptake) are dominant over these longer time domains (20). The sum of these data, therefore, support the hypothesis that a mucus clearance defect exists in CF, even in the setting of mild airway obstruction, but that this defect may be inhomogeneous and primarily localized to the small airway compartment at this stage of disease.

MCC measurements have now been used in a number of small clinical trials (Table 1). An acute stimulation of MCC was noted with several therapeutics that are now being developed for CF lung disease. These include hypertonic saline (HS) (16, 21), mannitol (17), amiloride (22), and uridine 5'-triphosphate (UTP) (12). HS and mannitol, both osmotic agents, appeared to yield the largest acute stimulation of clearance, whereas UTP only accelerated peripheral lung clearance rates in patients with $\mathrm{CF}$ when combined with amiloride. MCC was also measured during repetitive dosing of $\mathrm{HS}$ in patients with $\mathrm{CF}$, and demonstrated sustained improvements in clearance rates (i.e., $>8 \mathrm{~h}$ 
TABLE 1. MCC MEASUREMENTS IN CLINICAL TRIALS

\begin{tabular}{|c|c|c|c|c|}
\hline Drug & $\begin{array}{l}\text { Proposed Mechanism } \\
\text { of Action }\end{array}$ & Outcome & Investigator/Reference & Comments \\
\hline $\begin{array}{l}\text { Amiloride }(1 \mathrm{mM} \text {, } \\
\text { nebulized twice daily) } \\
\text { vs. saline; } \mathrm{n}=23(\mathrm{n}=6 \\
\text { treated } \times 3 \mathrm{wk})\end{array}$ & $\begin{array}{l}\text { Block sodium channels; } \\
\text { increase ASL volume }\end{array}$ & $\begin{array}{l}\text { Acutely increased MCC; } \\
\text { sustained improvement } \\
\text { after } 3 \mathrm{wk}\end{array}$ & App and colleagues (22) & $\begin{array}{l}\text { Nonsignificant effect of amiloride reported } \\
\text { by Bennett and colleagues in smaller } \\
\text { cohort (12) }\end{array}$ \\
\hline UTP $(n=14)$ & $\begin{array}{l}\mathrm{P} 2 \mathrm{Y}_{2} \text { agonist; stimulates } \\
\text { chloride secretion and } \\
\text { cilia beat frequency }\end{array}$ & $\begin{array}{l}\text { Increased peripheral lung } \\
\text { clearance in CF when } \\
\text { combined with } \\
\text { amiloride. }\end{array}$ & Bennett and colleagues (12) & $\begin{array}{l}\text { Peripheral clearance measured to avoid } \\
\text { confounding effects of higher C/P ratio } \\
\text { and increased cough in CF. }\end{array}$ \\
\hline $\begin{array}{l}\text { Hypertonic saline }(7 \% \\
\qquad \mathrm{NaCl} ; \mathrm{n}=10-24)\end{array}$ & $\begin{array}{l}\text { Increase ASL volume via } \\
\text { osmotic properties }\end{array}$ & $\begin{array}{l}\text { Acute and sustained effects } \\
\text { on MCC }\end{array}$ & $\begin{array}{l}\text { Robinson and colleagues } \\
\text { (21); Donaldson and } \\
\text { colleagues (16) }\end{array}$ & $\begin{array}{l}0.9-12.0 \% \mathrm{NaCl} \text { studied by Robinson and } \\
\text { colleagues }(21) ; 12 \% \text { saline was not } \\
\text { well tolerated }\end{array}$ \\
\hline $\begin{array}{l}\text { Mannitol (300 mg dry } \\
\text { powder inhaler; } \mathrm{n}=12 \text { ) }\end{array}$ & $\begin{array}{l}\text { Increase ASL volume via } \\
\text { osmotic properties }\end{array}$ & Acutely increased MCC & Robinson and colleagues (17) & $\begin{array}{l}\text { Mannitol compared to } 6 \% \mathrm{NaCl} \text {; yielded } \\
\text { similar changes in } \mathrm{MCC} \text { and } \mathrm{CC}\end{array}$ \\
\hline $\begin{array}{l}\text { Sodium 4-phenylbutyric } \\
\text { acid }(20 \mathrm{~g} / \mathrm{d} \text { by mouth } \\
\times 7 \mathrm{~d} ; \mathrm{n}=5)\end{array}$ & $\begin{array}{l}\text { Reduce } \Delta \mathrm{F} 508 \\
\text { degradation; stimulate } \\
\text { chloride transport }\end{array}$ & $\begin{array}{l}\text { No change in MCC or CC } \\
\text { from baseline after } 7 \mathrm{~d} \\
\text { of treatment }\end{array}$ & $\begin{array}{l}\text { Laube and Zeitlin } \\
\text { (unpublished data) }\end{array}$ & $\begin{array}{l}\text { Timing of MCC measurement may have } \\
\text { been inappropriate to detect any acute } \\
\text { effect of drug (i.e., }<7 \text { d). }\end{array}$ \\
\hline $\begin{array}{l}\text { Terbutaline (by inhalation; } \\
\qquad \mathrm{n}=10 \text { ) }\end{array}$ & $\begin{array}{l}\text { Increase ciliary beat } \\
\text { frequency in CF }\end{array}$ & $\begin{array}{l}\text { No acute effect on MCC } \\
\text { in CF }\end{array}$ & $\begin{array}{l}\text { Mortenson and colleagues } \\
(14,26)\end{array}$ & $\begin{array}{l}\text { Absent cAMP-mediated chloride secretion } \\
\text { in CF may limit effect; sample size } \\
\text { inadequate to detect subpopulation of } \\
\text { responders }\end{array}$ \\
\hline $\begin{array}{l}\text { Sodium bicarbonate }(1.5 \% \\
\text { solution } \times 5 \mathrm{ml} \text { by } \\
\text { inhalation; } \mathrm{n}=10)\end{array}$ & $\begin{array}{l}\text { Correct low pH ASL in CF, } \\
\text { which may increase cilia } \\
\text { beat frequency (34) }\end{array}$ & No acute effect on MCC & Corcoran and colleagues (27) & $\begin{array}{l}\text { Delivered dose may have been too low; } \\
\text { sample size inadequate to detect } \\
\text { subpopulation of responders }\end{array}$ \\
\hline $\begin{array}{l}\text { Pulmozyme }(2.5 \mathrm{mg} \text { twice } \\
\text { daily by inhalation } \times 6 \mathrm{~d} \text {; } \\
\mathrm{n}=10 \text { per treatment } \\
\text { group) }\end{array}$ & Reduce mucus viscosity & $\begin{array}{l}\text { No difference in MCC vs. } \\
\text { placebo after } 6 \mathrm{~d} \text { of } \\
\text { treatment }\end{array}$ & Laube and colleagues (28) & $\begin{array}{l}\text { Effect on CC is unknown since it was not } \\
\text { assessed }\end{array}$ \\
\hline $\begin{array}{l}\text { Moli1901 }(2.5 \mathrm{mg} \times 1 \text { dose } \\
\quad \text { by inhalation; } \mathrm{n}=3)\end{array}$ & $\begin{array}{l}\text { Stimulate chloride } \\
\text { transport (12) }\end{array}$ & $\begin{array}{l}\text { No change from baseline at } \\
24 \mathrm{~h} \text { after dosing }\end{array}$ & $\begin{array}{l}\text { Laube and Zeitlin } \\
\text { (unpublished data) }\end{array}$ & $\begin{array}{l}\text { Delivered dose may have been too low. } \\
\text { Timing of MCC measurement may } \\
\text { have been inappropriate to detect any } \\
\text { acute effect of drug (i.e., }<24 \text { h). }\end{array}$ \\
\hline
\end{tabular}

Definition of abbreviations: $\mathrm{ASL}=$ airway surface liquid; $\mathrm{CC}=$ cough clearance; $\mathrm{CF}=$ cystic fibrosis; $\mathrm{C} / \mathrm{P}=$ radioactive counts in central and peripheral lung zones; MCC $=$ mucociliary clearance; UTP $=$ uridine $5^{\prime}$-triphosphate.

after prior dose) within 2 weeks of initiating this therapy. In addition, an acute stimulation of clearance could still be observed at the end of the 2-week dosing period (i.e., no tachyphylaxis). Interestingly, the combination of amiloride and HS yielded acute, but not sustained, improvements in MCC, and did not improve lung function or symptoms, unlike HS alone, which did yield improvements in these clinical outcomes (16). Given that HS not only improved lung function, but also has been shown to reduce the frequency of pulmonary exacerbations (23), it is enticing to speculate that its sustained effect on MCC might in fact serve as a surrogate marker for improvement in one of these more traditional clinical outcomes. Clearly, though, this association will require additional study in order to validate a true causal link. Longer-acting and more potent analogs of UTP (denufosol tetrasodium; Inspire Pharmaceuticals, Inc., Durham, NC) and amiloride (PS 552-02; Parion Sciences, Inc., Durham, NC) are currently in development for CF, with the hope that these agents will also yield sustained improvements in MCC. Of note, PS 552-02 has been reported to improve MCC in normal subjects, and to have some evidence of pharmacodynamic durability (effect detectable at $4 \mathrm{~h}$ after dosing) (24), whereas the effects of denufosol on MCC in humans are not yet known.

In contrast to these agents that have been shown to accelerate MCC rates, a variety of other therapeutics that were predicted to improve MCC in CF have failed to show measurable effects. Although these failures may truly reflect a lack of efficacy of the study agent, it must also be considered that improved MCC measurement techniques and/or study designs might have uncovered beneficial effects. Therefore, it is instructive to examine these studies as well. Drugs proposed to reduce the degradation of the CF transmembrane conductance regulator protein (i.e., sodium 4-phenylbutyric acid [25]), increase ciliary beat frequency (i.e., terbutaline [26] and sodium bicarbonate [27]), decrease the viscosity of the mucus (Pulmozyme [28]; Genentech, Inc., South San Francisco, CA), or increase the ASL volume by stimulating chloride transport (Moli1901; Lantibio, Inc., Chapel Hill, NC) have been tested in patients with CF at different centers. Table 1 provides an outline of these studies, with potentially relevant shortcomings that prevented a positive outcome.

There are a number of possible explanations related to study design for the negative outcomes reported in these studies. First, the effect of a given drug may be short lived, and an effect on MCC may only be detected if the timing between dosing and MCC measurement is appropriate. If the pharmacodynamic properties of a drug are unknown, MCC assessments at acute and delayed time points after dosing may be required. Of note, the acute effects of sodium 4-phenylbutyric acid and Moli1901 on MCC were not assayed by nature of their respective study designs, and could account for the absence of any detectable effect on MCC. Second, as with any drug, an effective dose must be reached. This issue may be particularly problematic with inhaled therapies if formal drug delivery and pharmacokinetic information is not available before choosing a dose in "proof of mechanism" or therapeutic trials. Certainly the CF lung can be a particularly challenging target organ due to inhomogeneous ventilation/delivery and physical barriers posed to some drugs by a thickened mucus layer. Third, the MCC parameter being assessed should match the effect of the drug being delivered. For example, Pulmozyme may, in fact, alter sputum rheology in a fashion that predicts improved CC, but not cilia-dependent 
clearance (29). Therefore, to capture the effect of this drug on mucus clearance, a dedicated CC study might be necessary rather than the typical MCC study format, where cough is either not measured, or is measured after 60-90 minutes of measuring ciliadependent clearance. Similar problems could also be encountered if an agent primarily affects clearance from small versus large airways and the MCC study (i.e., the radiolabeled aerosol delivery strategy) is not designed to assess this airway region. Finally, a large number of patient- and study-related factors might increase the variability of MCC measurements or otherwise reduce the ability to detect meaningful differences affected by a novel therapy. For example, one must consider the other therapies being used by a patient, including those that might affect the baseline rate of MCC or CC (e.g., HS or rhDNase, antibiotic therapies), or alter the deposition of the study drug and/or radiotracer (e.g., bronchodilators). Holding potentially confounding medications or treatments (i.e., mechanical airway clearance maneuvers), or at least standardizing their timing before MCC measurement, must be considered carefully. Unwanted variability may also be encountered in longer trials, given the intrinsic variability of $\mathrm{CF}$ lung disease, thereby reducing study power and increasing necessary sample size to detect clearance rate changes in both crossover and parallel-group designs. Given these inherent problems, study designs that carefully consider these factors, and analysis plans that account for common confounders, are essential.

\section{FUTURE DIRECTIONS}

Despite the importance of being able to accurately quantitate $\mathrm{MCC} / \mathrm{CC}$ as an outcome for CF therapeutic trials, only a few $\mathrm{CF}$ centers have developed the capacity to perform these studies. Unfortunately, the techniques used to measure $\mathrm{MCC} / \mathrm{CC}$ at these centers are not standardized, making comparison of results difficult at times. Furthermore, with the limited use of MCC measures as an assessment of clinical efficacy, no rigorous assessments of intrasubject variability for any of the currently used methodologies have been made. Because an increasing number of drugs designed to improve $\mathrm{MCC} / \mathrm{CC}$ in $\mathrm{CF}$ are entering the therapeutic pipeline, it is vital to develop standard techniques for these measures in order to efficiently screen compounds at an early stage of development and rapidly advance those with the highest chance of being effective.

Perhaps the most important element to standardize of $\mathrm{MCC} /$ $\mathrm{CC}$ studies is the delivery of the radiolabeled aerosol. Because clearance rates are highly dependent on regional deposition of the radiolabeled particles in the lung, aerosol delivery to the bronchial airways must occur in a reproducible manner to minimize intra- and intersubject variability in particle deposition. However, it is primarily these delivery methods that differ between various study sites. In an effort to address this problem, aerosol delivery techniques that have been shown to improve the reproducibility of deposition are currently being optimized and simplified in a multicenter effort supported by the CF Foundation. These studies are also aimed at the standardization of data acquisition and image analysis protocols for assessing initial, regional deposition patterns, and the subsequent retention-versustime MCC data. Finally, because serial studies will be performed, data pertinent to within-subject repeatability and betweencenter reproducibility will be generated. This collaborative effort should significantly advance efforts to make MCC measurements comparable at $\mathrm{CF}$ centers that are currently active in this field.

The current methods for measuring MCC are designed to target delivery of radiolabeled particles to the bronchial airways, but the observation times and inhalation maneuvers are such that the measured $\mathrm{MCC}$ rates are primarily reflective of the largest of these airways. On the other hand, because the initial pulmonary pathophysiology in CF occurs in the small, peripheral conducting airways (30), it is likely that mucociliary dysfunction also begins there, as evidenced by two previously described comparisons of MCC in normal subjects and those with CF $(12,16)$. An inhalation method that has been employed to specifically target particle deposition to the small, bronchial airways is the extremely slow inhalation technique described by Anderson and colleagues (31). These investigators had subjects inhale large particles, $6-\mu \mathrm{m}$ mass median aerodynamic diameter, with very low inhalation flow rates $(0.04 \mathrm{~L} / \mathrm{s})$, conditions under which they predicted that these large particles would settle out in the small bronchial airways before reaching the alveolar region. The subsequent clearance kinetics that they measured through 96 hours after deposition supported this contention (i.e., a slow but significant clearance of particles from 24 to 96 hours, consistent with particle clearance from small airways. Incorporating such an inhalation method, along with retention measurements at later time points $(6-24 \mathrm{~h})$, into future studies may provide a better index of small airway clearance in CF for analysis of therapeutic effects in these airways. This may be particularly important for agents not expected to yield large, rapid ASL volume fluxes in central airways, and for drugs that preferentially affect small airways in $\mathrm{CF}$, as was seen with the combination of UTP and amiloride (12).

Although uncontrolled, spontaneous coughing can confound measures of MCC (32), more standardized, controlled measures of CC can be obtained by incorporating fixed numbers of voluntary coughs during the image acquisition period for assessing particle clearance $(32,33)$. Because certain agents may change sputum rheology in a manner that preferentially improves this mucus clearance parameter (vs. cilia-dependent clearance), $\mathrm{MCC} / \mathrm{CC}$ protocols that adequately measure $\mathrm{CC}$ are important. Of note, $\mathrm{MCC} / \mathrm{CC}$ studies that are primarily focused on the measurement of $\mathrm{CC}$ have not been performed in $\mathrm{CF}$, and previously used protocols have serious shortcomings in the assessment of this variable. The development of improved methodologies that incorporate standardized coughs at a time point closer to the delivery of radiolabel should be considered. Finally, spontaneous cough frequency, as a potential covariate in the analysis of $\mathrm{MCC} / \mathrm{CC}$, should also be recorded throughout the imaging period in all $\mathrm{MCC} / \mathrm{CC}$ studies.

Other aspects of MCC study designs should also be considered in relation to the study drug's proposed action. For example, if a drug is thought to have an immediate effect on $\mathrm{MCC} / \mathrm{CC}$, does not alter airway mechanics, but does elicit spontaneous coughing during administration, the study drug dosing should occur immediately before the inhalation of radioaerosol and subsequent $\gamma$ camera monitoring. On the other hand, if a drug acts acutely, but does alter airway mechanics (e.g., a bronchodilator) and doesn't elicit spontaneous cough, then dosing should occur immediately after inhalation of the radioaerosol to avoid differences in deposition pattern on drug and placebo study days. Finally, if a drug has a delayed onset while not affecting airway mechanics, then inhalation of radioaerosol should occur some time after inhalation of the drug (depending on peak effect or durability of the drug). As part of the preclinical trial development of new therapies to improve $\mathrm{MCC} / \mathrm{CC}$ in $\mathrm{CF}$, we recommend an acute, double-blinded, cross-over study design to first show biological effect. A positive outcome in such a trial may be followed by a longer design (e.g., 2 wk of repetitive dosing), similar to that recently performed for aerosolized HS (16). Such a trial allows detection of $\mathrm{MCC} / \mathrm{CC}$ changes (e.g., sustained effects on MCC rate) that might only occur with longer treatment 
periods, as well as comparison with other lung function endpoints.

\section{CONCLUSIONS}

The measurement of MCC and CC is a very promising, but incompletely developed, biomarker for $\mathrm{CF}$ clinical research. There is strong evidence that measurements of $\mathrm{MCC} / \mathrm{CC}$ are biologically and clinically relevant, in that they have been shown to reflect the underlying disease process and to be responsive to therapies aimed at normalizing ASL volume (e.g., HS, mannitol, amiloride, UTP). In addition, this outcome measure has the distinct advantage of being an index of both disease activity and drug effect in the critical target organ, the lung, as opposed to other outcome measures that might be used in drug trials of ion channel-directed therapies (e.g., nasal potential difference or sweat chloride). As a result, a positive effect on MCC testing might be more likely to translate into clinical benefit than a nonpulmonary outcome. Shortcomings that are currently being addressed include the lack of procedural standardization between centers that can perform MCC. This has limited the ability to compare results of studies performed at different centers, and, more importantly, has limited our ability to perform multicenter studies of new CF therapies using MCC as an outcome.

Once a standard operating procedure is refined and agreed upon, it will be important to assess the repeatability and reproducibility of these studies to facilitate the appropriate design of clinical trials. It is possible, however, that a single $\mathrm{MCC} / \mathrm{CC}$ protocol may not be adequate to address all aspects of mucus clearance, or be optimally suited for testing of all drugs in the development pipeline. Alternatively, several protocols could be created to assess different aspects of mucus transport (e.g., CC, small airway clearance), although each would need to be formally validated. Whether or not any particular aspect of an MCC assessment (e.g., a sustained effect on whole-lung clearance) will be shown to actually predict a clinically relevant outcome (e.g., reduced exacerbation frequency) - in other words, serve as a surrogate marker-is unclear, but would be an exciting development. Ultimately, dissemination of the ability to perform MCC measurements to other research centers should be feasible, and would greatly enhance the ability to test candidate CF therapies in a much more rapid and thorough fashion.

Conflict of Interest Statement: S.H.D. served as coinvestigator on research grants from Parion Sciences $(\$ 154,000$ in 2004) and Inspire Pharmaceuticals (pending 2007). T.E.C. does not have a financial relationship with a commercial entity that has an interest in the subject of this manuscript. B.L.L. received a research grant from Sepracor, Inc. (\$41,774 in 2004). W.D.B. was reimbursed in 2005 and 2006 by GlaxoSmithKline $(\$ 6,000$ total) for attending two scientific advisory board meetings. He is coinvestigator on research grants from Parion Sciences $(\$ 154,000$ in 2004) and Inspire Pharmaceuticals (pending 2007).

\section{References}

1. Rowe S, Accurso F, Clancy JP. Detection of CFTR activity in early phase clinical trials. Proc Am Thorac Soc 2007;4:387-398.

2. Donaldson SH, Boucher RC. Update on pathogenesis of cystic fibrosis lung disease. Curr Opin Pulm Med 2003;9:486-491.

3. Matsui H, Grubb B, Tarran R, Randell S, Gatzy J, Davis C, Boucher R. Evidence for periciliary liquid layer depletion, not abnormal ion composition, in the pathogenesis of cystic fibrosis airways disease. Cell 1998;95:1005-1015.

4. Matsui H, Verghese MW, Kesimer M, Schwab UE, Randell SH, Sheehan JK, Grubb BR, Boucher RC. Reduced three-dimensional motility in dehydrated airway mucus prevents neutrophil capture and killing bacteria on airway epithelial surfaces. J Immunol 2005;175:1090-1099.

5. Knowles MR, Boucher RC. Mucus clearance as a primary innate defense mechanism for mammalian airways. J Clin Invest 2002;109:571-577.
6. Saha G. Fundamentals of nuclear pharmacy, 5th ed. New York: Springer; 2004. p. 114.

7. Lay JC, Berry CR, Kim CS, Bennett WD. Retention of insoluble particles after local intrabronchial deposition in dogs. J Appl Physiol 1995;79: 1921-1929.

8. Alexis NE, Lay JC, Zeman KL, Geiser M, Kapp N, Bennett WD. In vivo particle uptake by airway macrophages in healthy volunteers. Am J Respir Cell Mol Biol 2006;34:305-313.

9. Smaldone GC, Palmer LB. Aerosolized antibiotics: current and future. Respir Care 2000;45:667-675.

10. Ilowite JS, Smaldone GC, Perry RJ, Bennett WD, Foster WM. Relationship between tracheobronchial particle clearance rates and sites of initial deposition in man. Arch Environ Health 1989;44:267-273.

11. Hofmann W, Sturm R. Stochastic model of particle clearance in human bronchial airways. J Aerosol Med 2004;17:73-89.

12. Bennett WD, Olivier KN, Zeman KL, Hohneker KW, Boucher RC, Knowles MR. Effect of uridine 5'-triphosphate plus amiloride on mucociliary clearance in adult cystic fibrosis. Am J Respir Crit Care Med 1996;153:1796-1801.

13. Robinson M, Eberl S, Tomlinson C, Daviskas E, Regnis JA, Bailey DL, Torzillo PJ, Menache M, Bye PT. Regional mucociliary clearance in patients with cystic fibrosis. J Aerosol Med 2000;13:73-86.

14. Mortensen J, Lange P, Nyboe J, Groth S. Lung mucociliary clearance. Eur J Nucl Med 1994;21:953-961.

15. Kuni C, duCret RP. Manual of nuclear medicine imaging. New York: Thieme; 1997. p. 244.

16. Donaldson SH, Bennett WD, Zeman KL, Knowles MR, Tarran R, Boucher RC. Mucus clearance and lung function in cystic fibrosis with hypertonic saline. N Engl J Med 2006;354:241-250.

17. Robinson M, Daviskas E, Eberl S, Baker J, Chan HK, Anderson SD, Bye PT. The effect of inhaled mannitol on bronchial mucus clearance in cystic fibrosis patients: a pilot study. Eur Respir J 1999;14:678-685.

18. Smaldone GC, Perry R, Bennett WD, Messina M, Zwang J, Ilowite J. Interpretation of $24 \mathrm{hr}$ lung retention in studies of mucociliary clearance. J Aerosol Med 1988;1:11-20.

19. Regnis JA, Zeman KL, Noone PG, Knowles MR, Bennett WD. Prolonged airway retention of insoluble particles in cystic fibrosis versus primary ciliary dyskinesia. Exp Lung Res 2000;26:149-162.

20. Lindstrom M, Camner P, Falk R, Hjelte L, Philipson K, Svartengren M. Long-term clearance from small airways in patients with cystic fibrosis. Eur Respir J 2005;25:317-323.

21. Robinson M, Hemming AL, Regnis JA, Wong AG, Bailey DL, Bautovich GJ, King M, Bye PT. Effect of increasing doses of hypertonic saline on mucociliary clearance in patients with cystic fibrosis. Thorax 1997; 52:900-903.

22. App E, King M, Helfesrieder R, Kohler D, Matthys H. Acute and longterm amiloride inhalation in cystic fibrosis lung disease: a rational approach to cystic fibrosis therapy. Am Rev Respir Dis 1990;141:605612.

23. Elkins MR, Robinson M, Rose BR, Harbour C, Moriarty CP, Marks GB, Belousova EG, Xuan W, Bye PT. A controlled trial of long-term inhaled hypertonic saline in patients with cystic fibrosis. $N$ Engl J Med 2006;354:229-240.

24. Donaldson S, Smith R, Doran J, DiMassimo B, Zeman K, Bennett B, Hurd H, Hopkins S. Safety, pharmacokinetics and effects on mucus clearance following adminsitration of 552-02 to normal healthy volunteers [abstract]. Pediatr Pulmonol Suppl 2005;28:218.

25. Rubenstein RC, Zeitlin PL. A pilot clinical trial of oral sodium 4-phenylbutyrate (Buphenyl) in $\Delta$ F508-homozygous cystic fibrosis patients: partial restoration of nasal epithelial CFTR function. Am J Respir Crit Care Med 1998;157:484-490.

26. Mortensen J, Hansen A, Falk M, Nielsen IK, Groth S. Reduced effect of inhaled $\beta$ 2-adrenergic agonists on lung mucociliary clearance in patients with cystic fibrosis. Chest 1993;103:805-811.

27. Corcoran TE, Kreindler JL, Hartigan E, Hurban S, Marcinkowksi A, Gushchin A, Orenstein D, Pilewski JM. Nebulized sodium bicarbonate and mucociliary clearance in cystic fibrosis [abstract]. Proc Am Thorac Soc 2006;3:A727.

28. Laube BL, Auci RM, Shields DE, Christiansen DH, Lucas MK, Fuchs HJ, Rosenstein BJ. Effect of rhDNase on airflow obstruction and mucociliary clearance in cystic fibrosis. Am J Respir Crit Care Med 1996; 153:752-760.

29. King M, Dasgupta B, Tomkiewicz RP, Brown NE. Rheology of cystic fibrosis sputum after in vitro treatment with hypertonic saline alone 
and in combination with recombinant human deoxyribonuclease I. Am J Respir Crit Care Med 1997;156:173-177.

30. Davis PB. Cystic fibrosis. New York: Marcel Dekker; 1993.

31. Anderson M, Philipson K, Svartengren M, Camner P. Human deposition and clearance of 6-micron particles inhaled with an extremely low flow rate. Exp Lung Res 1995;21:187-195.

32. Robinson M, Regnis JA, Bailey DL, King M, Bautovich GJ, Bye PT. Effect of hypertonic saline, amiloride, and cough on mucociliary clear- ance in patients with cystic fibrosis. Am J Respir Crit Care Med 1996; 153:1503-1509.

33. Bennett WD, Chapman WF, Mascarella JM. The acute effect of ipratropium bromide bronchodilator therapy on cough clearance in COPD. Chest 1993;103:488-495.

34. Tate S, MacGregor G, Davis M, Innes JA, Greening AP. Airways in cystic fibrosis are acidified: detection by exhaled breath condensate. Thorax 2002;57:926-929. 